\title{
Preclinical Trial of Propolis Extract in Prevention of High Salt Diet- Induced Hypertension
}

\author{
Ade Heri Mulyati ${ }^{1,2}$, Ahmad Sulaeman ${ }^{1, *}$, Sri Anna Marliyati ${ }^{1}$, Mohamad Rafi ${ }^{3}$, Al Mukhlas Fikri ${ }^{1}$
}

Ade Heri Mulyati ${ }^{1,2}$, Ahmad

Sulaeman ${ }^{1, *}$, Sri Anna Marliyati',

Mohamad Rafi ${ }^{3}$, Al Mukhlas Fikri'

'Department of Community Nutrition, Faculty of Human Ecology, IPB University, Bogor 16680, INDONESIA.

${ }^{2}$ Department of Chemistry, Faculty of Mathematics and Natural Sciences, University of Pakuan, Bogor 16163, INDONESIA.

${ }^{3}$ Department of Chemistry, Faculty of Mathematics and Natural Sciences, IPB

University, Bogor 16680, INDONESIA

\section{Correspondence}

\section{Ahmad Sulaeman}

Department of Community Nutrition, Faculty of Human Ecology, IPB University, Bogor 16680, INDONESIA.

E-mail: asulaema06@gmail.com

\section{History}

- Submission Date: 15-10-2020;

- Review completed: 01-11-2020;

- Accepted Date: 13-11-2020.

DOI : 10.5530/pj.2021.13.13

Article Available online

http://www.phcogj.com/v13/i1

\section{Copyright}

(C) 2021 Phcogi.Com. This is an openaccess article distributed under the terms of the Creative Commons Attribution 4.0 International license.

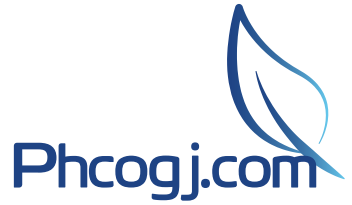

\section{ABSTRACT}

Background: Propolis has been widely reported as having various biological activities However, Indonesian propolis seems to be less explored. Objective: The present study aimed to analyze the antihypertensive activity of Indonesian propolis in rats. Materials and Methods: Hypertension was induced by high- $\mathrm{NaCl}(8 \%)$ diet for 3 weeks. A total of 36 rats were divided into 6 groups, including standard diet group (SD), high- $\mathrm{NaCl}$ diet group ( $\mathrm{NaD}$ ), high- $\mathrm{NaCl}$ diet group + captopril $(25 \mathrm{mg} / \mathrm{kg}$ ) (PD), high- $\mathrm{NaCl}$ diet + propolis from Riau Archipelago (NaDP1), high- $\mathrm{NaCl}$ diet + propolis from Lampung ( $\mathrm{NaDP} 2$ ) and high- $\mathrm{NaCl}$ diet + propolis from South Sulawesi (NaDP3). Propolis was daily administered at dose of $200 \mathrm{mg} / \mathrm{kg}$ on hypertensive rats for 1 week. Blood pressure and body weight were weekly measured. Moreover, routine urine analysis, haematological parameters and lipid profiles at week 4 were determined. Results: The results showed that high- $\mathrm{NaCl}$ diet successfully induced hypertension in rats after 3 weeks of intervention. However, the diet did not cause weight gain $(p>0.05)$. All Indonesian propolis samples significantly reversed either systolic or diastolic blood pressure of hypertensive rats. From urine analysis, propolis from Riau Archipelago and Lampung showed diuretic effect. The haematological analysis mainly showed no significant difference compared standard diet group. Furthermore, LDL and HDL concentrations were significantly improved by propolis from Lampung and South Sulawesi, respectively $(\mathrm{p}<0.05)$. In addition, we only found significant decrease in relative weight of liver in all groups administered with high- $\mathrm{NaCl}$ diet $(p<0.05)$. Conclusion: The present study suggests that all Indonesian propolis possessed antihypertensive activity.

Key words: Antihypertension, Blood pressure, High- $\mathrm{NaCl}$ diet, Stingless bee propolis.

\section{INTRODUCTION}

Hypertension is a serious public health problem and the major cause of premature death worldwide. ${ }^{1}$ WHO (2019) reported around 1.13 billion people had hypertension and mostly lived in low-middle income countries. Hypertension is risk factor for several diseases, including heart attack, stroke, kidney damage, diabetes and even cancer. ${ }^{3-6}$ In addition, hypertension also may reduce cognitive performance and cause economic lost in either micro or macro scale. ${ }^{7-8}$

Prevention of hypertension by controlling modifiable risk factors would have beneficial effect on the public health. ${ }^{9}$ High intake of salty food is one of the important risk factor for developing hypertension. ${ }^{10-12}$ The estimated salt intake is about 9-12 g per day in most countries which is higher than WHO recommendation of less than 5 g. ${ }^{11}$ High salt intake causes the impairment of renin angiotensin-aldosterone (RAA) system and oxidative stress leading to the elevation of blood pressure. ${ }^{13}$

The management of hypertension is currently by pharmaceutical therapies, however, it is costly, has adverse effect, and requires medical intervention. ${ }^{9}$ The triple therapies, including diuretics, a calcium channel blocker and an angiotensinconverting enzyme inhibitor/angiotensin receptor blocker, cannot reduce the risk for resistant hypertension due to high salt intake. ${ }^{13}$ In addition, the current management seems to be not effective in controlling hypertension, thus it needs another therapies, including dietary, lifestyle and nutritional supplement strategies..$^{14}$ Meanwhile, the study on bioactive natural components has been increased describing the mechanism of action in the prevention of hypertension. ${ }^{15}$

Propolis is a natural product manufactured by bee from plant resins. ${ }^{16}$ The biological activities of propolis have been widely studied, namely antioxidant, antibacterial, antiviral, antifungal, anti-inflammatory, anticancer, antidiabetes, immunomodulatory, antiemetic properties, and also to show antihypertensive property. ${ }^{16-20}$ The previous research showed that the constituents of Brazilian propolis ameliorated the hypertension in rats. $^{21}$ However, there are some challenges on propolis research due to its diverse chemical constituents which depends on botanical origin, bee species and the preparation of extract. ${ }^{15}$ Therefore, each region possibly has their unique properties.

Indonesia is a country with high biodiversity which has the potential to produce a great variety of propolis. Several studies on Indonesian propolis have showed that Indonesia propolis possessed antioxidant, immunomodulatory, anti-inflammatory, and anticancer activities. ${ }^{20,22-24}$ However, there has

Cite this article: Mulyati $\mathrm{AH}$, Sulaeman A, Marliyati SA, Rafi M, Fikri AM. Preclinical Trial of Propolis Extract in Prevention of High Salt Diet-Induced Hypertension. Pharmacog J. 2021;13(1): 89-96. 
not been study to investigate the antihypertensive activity of Indonesian propolis. Therefore, this study aimed to analyze the effect of Indonesian propolis from 3 provinces of Indonesia on $\mathrm{NaCl}$-induced hypertension in rats.

\section{MATERIALS AND METHODS}

\section{Preparation of propolis extracts}

The raw propolis samples were collected from Riau Archipelago, Lampung, and South Sulawesi in 2018. The extraction of raw samples was performed using ultrasound-assisted extraction. The samples were grounded into small pieces $(0.5-1 \mathrm{~cm})$ and dissolved in $70 \%$ ethanol for $24 \mathrm{~h}(1: 10)$. Furthermore, the mixtures were filtered using Whatman No 1 fitler paper. The residues were then re-dissolved in $70 \%$ ethanol following the previous procedures. The filtrate from the $1^{\text {st }}$ and $2^{\text {nd }}$ extraction were combined. The combined filtrates were kept for 1 day to precipitate the wax and subsequently evaporated to obtain dry extracts. The dry extracts were kept under $-20^{\circ} \mathrm{C}$ until use. ${ }^{20}$

\section{Animals and diets}

A total of 36 Sprague Dawley rats (all 250-300 g; 7-8 weeks of age) were obtained from Biopharmaca Research Center, IPB University, Bogor, West Java. The hypertension was induced by sodium chloride $(\mathrm{NaCl})$ (Sigma-Aldrich, Germany)-enriched diet for 3 weeks. The animals were divided into 6 groups $(\mathrm{n}=6)$, namely standard diet group (SD), high (8\%) $\mathrm{NaCl}$ diet group $(\mathrm{NaD})$, high (8\%) $\mathrm{NaCl}$ diet group + captopril (25 $\mathrm{mg} / \mathrm{kg}$ ) or positive control group (PD), high $(8 \%) \mathrm{NaCl}$ diet + propolis from Riau Archipelago (NaDP1), high (8\%) $\mathrm{NaCl}$ diet + propolis from Lampung (NaDP2), high (8\%) $\mathrm{NaCl}$ diet + propolis from South Sulawesi (NaDP3). NaD has been reported to induce hypertension in rats for 3 weeks. ${ }^{25-27}$ All propolis samples were given at dose of $200 \mathrm{mg} /$ $\mathrm{kg}$. The prior literatures showed that the administration of propolis at that dose inhibited tyrosine hydroxylase activity and reduced oxidative stress on chemically-induced hypertension in rats. ${ }^{28-29}$ The animals were acclimatized for 2 weeks and kept under standard laboratory condition (temperature of $22-25^{\circ} \mathrm{C}$; $12: 12$ light: dark cycles).

The rats were categorized to have hypertension when systolic blood pressure $(\mathrm{SBP}) \geq 140 \mathrm{mmHg} \cdot{ }^{29-30}$ The hypertensive rats then administered with propolis for 1 week, while high- $\mathrm{NaCl}$ diet was continued. Body weight and blood pressure was weekly measured from week 0 until week 4 . In addition, the rats were euthanized at week 4 to collect urine and blood samples for analysis. Heart, liver, kidney, spleen, lungs and urinary bladder were harvested to measure the weight. All experimental procedures have been approved by the Animal Care and Use Committee, IPB University (No. 176-2020 IPB).

\section{Blood pressure measurement and urine collection}

The procedures of blood pressure measurement referred to Malkoff et al. with slight modification. The blood pressure was weekly measured through a tail cuff in the morning using non-invasive blood pressure system (CODA, Kent Scientific, USA). The animals were placed in a holder for acclimatization for 10-15 minutes, whereas room temperature was kept at $26^{\circ} \mathrm{C}$. Blood pressure was read 10 times and the means were determined. ${ }^{31}$

\section{Biochemical analysis}

Blood lipid profiles, including triglyceride, cholesterol, high-density lipoprotein (HDL) and low-density lipoprotein (LDL), were determined using Cholesterol Oxidase-Phenol Aminophenazone (CHOD-PAP) standard methods. Blood samples were also used to analyse the routine haematological analysis on an Auto Hematology analyzer (Mindray BC-2800Vet, Shenzhen Mindray Bio-Medica; Electronics Co., Ltd, China). ${ }^{32}$ The haematological analysis included white blood cells
(WBC), lymphocytes, monocytes, granulocytes, red blood cells (RBC), haemoglobin $(\mathrm{Hb})$, haematocrits (Hct), mean corpuscular volume $(\mathrm{MCV})$, mean corpuscular haemoglobin $(\mathrm{MCH})$, mean corpuscular haemoglobin concentration (MCHC), red cell distribution width (RDW), platelets (PLT), mean platelet volume (MPV). Meanwhile, the urine volume was collected for $24 \mathrm{~h}$ at the last day of intervention. The quantitative ( $\mathrm{pH}$, glucose, and urine density) and qualitative (colour, consistency, leukocytes, nitrite, protein, ketone bodies, urobilinogen, bilirubin, erythrocytes and $\mathrm{Hb}$ ) analysis of urine samples were performed using urinalysis reagent strips (Helath Mate CYBOW REF 0004 reagent strips for Urynalisis. IVD. DFI Co.Ltd)

\section{Statistical analysis}

Data were expressed as mean \pm standard deviation. The differences between groups were analysed with ANOVA followed by post-hoc Tukey's test. The significance was considered at p-value $<0.05$.

\section{RESULTS}

To confirm whether high- $\mathrm{NaCl}$ consumption affects body weight, we weekly measured the weight of animals. The weight changes during the administration of high- $\mathrm{NaCl}$ diet and propolis are shown in Table 1. However, the weight of animals during administration of a high- $\mathrm{NaCl}$ diet for 3 weeks did not change statistically $(p>0.05)$. After propolis administration for 1 week, the weight of animals was also not affected ( $\mathrm{p}>0.05)$.

A high- $\mathrm{NaCl}$ diet successfully caused hypertension in rats after 3 weeks of intervention. Either systolic or diastolic blood pressure was statistically raised at week 3 in all high- $\mathrm{NaCl}$ groups compared to standard diet group $(\mathrm{P}<0.05)$. All propolis samples remarkably reduced SBP and DBP compared to high- $\mathrm{NaCl}$ only group after 1 week of administration $(p<0.05)$. The biggest changes were found in groups administered with propolis from Riau Archipelago and South Sulawesi, where the effect seemed to be similar with positive control group. The blood pressure of animal during the treatments can be seen in Table 2 and Table 3.

Result of urine test showed that propolis seems to increase the 24-h urine volume. Propolis from Riau Archipelago and Lampung increased significantly the 24-h urine volume compared to standard diet group $(\mathrm{p}<0.05)$. It was confirmed by lighter urine colour in both groups. However, the other parameters (density, $\mathrm{pH}$, consistency, leukocytes, nitrite, glucose, ketone, urobilinogen, bilirubin, erythrocytes, and haemoglobin) showed not statistically significant difference. Nevertheless, protein were relatively higher in all propolis groups. The result of urine test can be seen in Table 4 .

Table 5 shows lipid profiles of rats at the end of intervention. The present study found no differences in triglyceride and cholesterol concentrations between the groups. However, among the groups with high- $\mathrm{NaCl}$ diet, LDL concentrations were significantly decreased in group administered with propolis from Lampung compared to high- $\mathrm{NaCl}$ only group $(\mathrm{p}<0.05)$. In addition, HDL concentrations significantly increased in group administered with propolis from South Sulawesi compared to standard diet $(\mathrm{p}<0.05)$.

Result of haematological analysis is presented in Table 6. Almost all haematological parameters did not differ compared to standard diet group, except lymphocytes levels. The concentration of lymphocytes increased significantly in several groups, including positive control group, group administered with propolis from Riau Archipelago and Lampung compared to standard diet group. Nevertheless, we found relatively low levels of WBC, monocytes, granulocytes, RBC, haemoglobin, HCT, MCHC, PLT and PCT in high- $\mathrm{NaCl}$ only group compared to propolis group(s).

The weight of organs was measured to see the possibility of pathological 
Table 1: Weight of rats pre and post-treatment (gram).

\begin{tabular}{|c|c|c|c|c|c|c|c|}
\hline Group & Week 0 & Week 1 & Week 2 & Week 3 & Week 4 & Weight diference $^{1}$ & Weight difference ${ }^{2}$ \\
\hline Standard diet & $324.29 \pm 27.99$ & $311.50 \pm 18.19$ & $322.79 \pm 9.15$ & $315.93 \pm 6.92$ & $303.21 \pm 12.53$ & $-8.36 \pm 25.12$ & $-12.71 \pm 13.97$ \\
\hline High- $\mathrm{NaCl}$ diet group & $292.40 \pm 32.02$ & $259.17 \pm 37.21$ & $280.60 \pm 38.28$ & $284.90 \pm 31.71$ & $287.40 \pm 29.38$ & $-7.50 \pm 10.46$ & $2.50 \pm 14.26$ \\
\hline High- $\mathrm{NaCl}$ diet + captopril & $313.30 \pm 27.24$ & $304.83 \pm 21.48$ & $327.08 \pm 9.90$ & $320.00 \pm 8.62$ & $327.80 \pm 22.12$ & $6.70 \pm 25.04$ & $7.80 \pm 18.14$ \\
\hline $\begin{array}{l}\text { High- } \mathrm{NaCl} \text { diet }+ \text { propolis from } \\
\text { Riau Archipelago }\end{array}$ & $282.70 \pm 15.50$ & $281.67 \pm 17.37$ & $297.67 \pm 21.41$ & $287.30 \pm 12.98$ & $289.70 \pm 12.11$ & $4.60 \pm 12.19$ & $2.40 \pm 16.15$ \\
\hline $\begin{array}{l}\text { High- } \mathrm{NaCl} \text { diet + propolis from } \\
\text { Lampung }\end{array}$ & $287.17 \pm 24.82$ & $287.08 \pm 22.16$ & $303.92 \pm 23.57$ & $303.75 \pm 22.26$ & $309.75 \pm 35.86$ & $16.58 \pm 6.66$ & $6.00 \pm 20.09$ \\
\hline $\begin{array}{l}\text { High- } \mathrm{NaCl} \text { diet }+ \text { propolis from } \\
\text { South Sulawesi }\end{array}$ & $265.70 \pm 27.73$ & $271.58 \pm 16.38$ & $281.67 \pm 19.97$ & $277.10 \pm 17.95$ & $278.40 \pm 26.90$ & $11.40 \pm 13.25$ & $1.30 \pm 10.73$ \\
\hline
\end{tabular}

Data are mean \pm standard deviation $(n=6)$

${ }^{1}$ Weight difference $=$ weight at week 3 - weight at week 0

${ }^{2}$ Weight difference $=$ weight at week $4-$ weigh at week 3

Table 2: Systolic blood pressure $(\mathrm{mmHg})$ during the treatment.

\begin{tabular}{|c|c|c|c|c|c|c|}
\hline Group & Week 0 & Week 1 & Week 2 & Week 3 & Week 4 & SBP Difference ${ }^{*}$ \\
\hline Standard diet & $112.85 \pm 17.58^{\mathrm{a}}$ & $129.68 \pm 17.45^{c}$ & $121.72 \pm 19.78^{b}$ & $134.36 \pm 26.28^{\mathrm{c}}$ & $127.19 \pm 11.95^{\mathrm{b}}$ & $-7.17 \pm 31.64^{\mathrm{a}, \mathrm{b}}$ \\
\hline High- $\mathrm{NaCl}$ diet group & $121.71 \pm 23.31^{\mathrm{a}}$ & $155.70 \pm 25.93^{\mathrm{a}}$ & $138.40 \pm 21.18^{\mathrm{a}}$ & $162.00 \pm 10.46^{\mathrm{b}}$ & $161.85 \pm 21.38^{\mathrm{a}}$ & $-0.15 \pm 24.69^{\mathrm{a}}$ \\
\hline High- $\mathrm{NaCl}$ diet + captopril & $120.10 \pm 14.01^{\mathrm{a}}$ & $150.80 \pm 20.48^{\mathrm{a}, \mathrm{b}}$ & $140.46 \pm 16.94^{\mathrm{a}}$ & $159.25 \pm 10.92^{\mathrm{b}}$ & $127.61 \pm 14.59^{b}$ & $-31.64 \pm 19.14^{c, \mathrm{~d}}$ \\
\hline High- $\mathrm{NaCl}$ diet + propolis from Riau Archipelago & $118.66 \pm 20.88^{\mathrm{a}}$ & $146.23 \pm 13.08^{\mathrm{a}, \mathrm{b}}$ & $149.19 \pm 22.28^{\mathrm{a}}$ & $177.81 \pm 13.25^{\mathrm{a}}$ & $133.69 \pm 14.33^{\mathrm{b}}$ & $-44.12 \pm 20.11^{\mathrm{d}}$ \\
\hline High- $\mathrm{NaCl}$ diet + propolis from Lampung & $116.82 \pm 16.86^{\mathrm{a}}$ & $139.91 \pm 20.88^{\mathrm{b}, \mathrm{c}}$ & $147.45 \pm 27.44^{\mathrm{a}}$ & $156.64 \pm 22.06^{\mathrm{b}}$ & $135.44 \pm 8.93^{\mathrm{b}}$ & $-21.20 \pm 26.01^{b, c}$ \\
\hline High- $\mathrm{NaCl}$ diet + propolis from South Sulawesi & $112.12 \pm 14.36^{\mathrm{a}}$ & $144.91 \pm 27.64^{\mathrm{a}, \mathrm{b}}$ & $146.31 \pm 28.59^{\mathrm{a}}$ & $165.93 \pm 20.40^{\mathrm{b}}$ & $133.03 \pm 15.09^{b}$ & $-32.90 \pm 21.68^{d}$ \\
\hline
\end{tabular}

Data are mean \pm standard deviation $(n=6)$

The different superscript in the same column are statistically significant $(p<0.05)$

${ }^{\star}$ Systolic blood pressure (SBP) difference $=$ SBP at week $4-$ SBP at week 3

Table 3: Diastolic blood pressure $(\mathbf{m m H g})$ during the treatment.

\begin{tabular}{|c|c|c|c|c|c|c|}
\hline Group & Week 0 & Week 1 & Week 2 & Week 3 & Week 4 & DBP Difference* \\
\hline Standard diet & $90.63 \pm 16.38^{\mathrm{a}}$ & $99.85 \pm 12.76^{\mathrm{b}}$ & $92.54 \pm 14.83^{\mathrm{b}}$ & $101.20 \pm 21.25^{\mathrm{c}}$ & $92.29 \pm 11.60^{c}$ & $-8.91 \pm 24.41^{\mathrm{b}, \mathrm{c}}$ \\
\hline High- $\mathrm{NaCl}$ diet group & $94.46 \pm 28.46^{\mathrm{a}}$ & $116.11 \pm 29.17^{\mathrm{a}}$ & $96.31 \pm 20.43^{\mathrm{a}, \mathrm{b}}$ & $119.85 \pm 13.27^{\mathrm{a}, \mathrm{b}}$ & $123.17 \pm 18.62^{\mathrm{a}}$ & $3.33 \pm 19.64^{\mathrm{a}}$ \\
\hline High- $\mathrm{NaCl}$ diet + captopril & $96.20 \pm 10.79^{\mathrm{a}}$ & $111.32 \pm 20.37^{\mathrm{a}, \mathrm{b}}$ & $108.31 \pm 15.79^{\mathrm{a}, \mathrm{b}}$ & $118.66 \pm 11.00^{\mathrm{b}}$ & $91.35 \pm 13.45^{c}$ & $27.31 \pm 18.78^{\mathrm{c}, \mathrm{d}}$ \\
\hline High- $\mathrm{NaCl}$ diet + propolis from Riau Archipelago & $93.34 \pm 20.08^{\mathrm{a}}$ & $105.75 \pm 11.51^{\mathrm{a}, \mathrm{b}}$ & $109.14 \pm 16.42^{\mathrm{a}}$ & $130.76 \pm 13.21^{\mathrm{a}}$ & $100.33 \pm 11.43^{b, c}$ & $-30.43 \pm 14.45^{\mathrm{d}}$ \\
\hline High- $\mathrm{NaCl}$ diet + propolis from Lampung & $97.08 \pm 17.43^{\mathrm{a}}$ & $111.11 \pm 17.14^{\mathrm{a}, \mathrm{b}}$ & $106.66 \pm 24.22^{\mathrm{a}, \mathrm{b}}$ & $116.47 \pm 23.60^{\mathrm{b}}$ & $102.24 \pm 11.27^{\mathrm{b}}$ & $-14.23 \pm 23.67^{b}$ \\
\hline High- $\mathrm{NaCl}$ diet + propolis from South Sulawesi & $90.92 \pm 18.73^{\mathrm{a}}$ & $109.60 \pm 26.96^{\mathrm{a}, \mathrm{b}}$ & $115.94 \pm 27.10^{\mathrm{a}}$ & $125.72 \pm 19.24^{\mathrm{a}, \mathrm{b}}$ & $96.23 \pm 15.06^{\mathrm{b}, \mathrm{c}}$ & $-29.49 \pm 20.27^{\mathrm{d}}$ \\
\hline
\end{tabular}

Data ara mean \pm standard deviation $(n=6)$

Diastolic blood pressure (DBP) difference $=$ DBP at week $4-$ DBP at week 3

The different superscript in the same line are statistically significant $(\mathrm{p}<0.05)$

\section{Table 4: Lipid Profile.}

\begin{tabular}{|c|c|c|c|c|}
\hline Groups & $\begin{array}{l}\text { Triglycerides } \\
\text { (mg/ dL) }\end{array}$ & $\begin{array}{l}\text { Cholesterol } \\
(\mathrm{mg} / \mathrm{dL})\end{array}$ & $\mathrm{HDL}(\mathrm{mg} / \mathrm{dL})$ & $\mathrm{LDL}(\mathrm{mg} / \mathrm{dL})$ \\
\hline Standard diet & $97,30 \pm 13,95^{\mathrm{a}, \mathrm{b}}$ & $84,99 \pm 9,71^{\mathrm{a}, \mathrm{b}}$ & $27,70 \pm 2,67^{\mathrm{b}}$ & $59,99 \pm 8,57^{\mathrm{a}, \mathrm{b}}$ \\
\hline High- $\mathrm{NaCl}$ diet group & $92,63 \pm 9,17^{\mathrm{a}, \mathrm{b}}$ & $93,43 \pm 12,54^{\mathrm{a}, \mathrm{b}}$ & $29,09 \pm 5,76^{\mathrm{a}, \mathrm{b}}$ & $69,09 \pm 10,37^{\mathrm{a}}$ \\
\hline High- $\mathrm{NaCl}$ diet + captopril & $106,82 \pm 22,83^{\mathrm{a}, \mathrm{b}}$ & $72,37 \pm 11,11^{\mathrm{b}}$ & $25,83 \pm 3,34^{\mathrm{b}}$ & $45,84 \pm 7,62^{\mathrm{b}}$ \\
\hline High- $\mathrm{NaCl}$ diet + propolis from Riau Archipelago & $82,48 \pm 5,88^{b}$ & $77,45 \pm 3,81^{\mathrm{b}}$ & $29,10 \pm 3,20^{\mathrm{a}, \mathrm{b}}$ & $55,12 \pm 4,33^{\mathrm{a}, \mathrm{b}}$ \\
\hline High- $\mathrm{NaCl}$ diet + propolis from Lampung & $112,36 \pm 13,04^{\mathrm{a}}$ & $78,27 \pm 8,31^{b}$ & $27,23 \pm 3,65^{\mathrm{b}}$ & $50,35 \pm 7,04^{\mathrm{b}}$ \\
\hline High- $\mathrm{NaCl}$ diet + propolis from South Sulawesi & $96,81 \pm 6,54^{\mathrm{a}, \mathrm{b}}$ & $105,38 \pm 18,97^{a}$ & $36,06 \pm 5,67^{a}$ & $70,56 \pm 6,65^{\mathrm{a}}$ \\
\hline
\end{tabular}

changes. Relative weight of organs is depicted in Table 7. Heart seemed to be altered during the treatments. Decreased relative weight of heart was found in all groups administered with high- $\mathrm{NaCl}$. Meanwhile, the relative weight of liver, spleen, lungs and urinary bladder did not differ significantly between the groups.

\section{DISCUSSION}

Obesity has been confirmed as one of the main risk factors for developing hypertension. ${ }^{33-34}$ In the other hand, high consumption of salty food is strongly associated with obesity and probably due to increase in leptin production, leptin resistance and endogenous fructose production. ${ }^{35-36}$ However, the present study found no significantly change in weight of animals after high- $\mathrm{NaCl}$ diet intervention and propolis administration. Consumption of salty diet is associated with osmotic stress and will be compensated with increase in water consumption which may limit food intake. ${ }^{37}$ The present result is also in line with the prior study 
Table 5: Urine profile.

\begin{tabular}{|c|c|c|c|c|c|c|}
\hline \multirow{2}{*}{ Parameters } & \multicolumn{6}{|c|}{ Groups } \\
\hline & SD & $\mathrm{NaD}$ & PD & NaDP1 & $\mathrm{NaDP} 2$ & NaDP3 \\
\hline $24 \mathrm{~h}$ urine volume & $14.42 \pm 2.95^{\mathrm{c}}$ & $18.42 \pm 4.17^{\mathrm{b}, \mathrm{c}}$ & $13.38 \pm 2.05^{\mathrm{c}}$ & $34.67 \pm 13.26^{\mathrm{a}}$ & $29.67 \pm 10.84^{\mathrm{a}, \mathrm{b}}$ & $21.04 \pm 4.53^{\mathrm{b}, \mathrm{c}}$ \\
\hline Density & $1002.14 \pm 2.67$ & $1004.17 \pm 3.76$ & $1005.00 \pm 3.16$ & $1005.00 \pm 0.00$ & $1005.00 \pm 2.58$ & $1004.00 \pm 2.24$ \\
\hline $\mathrm{Ph}$ & $8.14 \pm 0.24$ & $8.00 \pm 0.32$ & $8.00 \pm 0.32$ & $8.10 \pm 0.22$ & $8.17 \pm 0.26$ & $7.90 \pm 0.22$ \\
\hline Color & Light brown & Light brown & Yellowish-light brown & Yellowish-light brown & Yellowish-light brown & Light brown \\
\hline Consistency & Thin & Thin & Thin & Thin & Thin & Thin \\
\hline Leukocytes $(\mathrm{leu} / \mu \mathrm{l})$ & Negative & Negative & Negative & Negative & Negative & Negative \\
\hline Nitrite & Negative & Negative & Negative & Negative & Negative & Negative \\
\hline Protein & $(+)-(+++)$ & $(+)-(++)$ & $(++)-(++++)$ & $(+++)-(++++)$ & $(+++)-(++++)$ & $(+++)-(++++)$ \\
\hline Glucose (mg/dl) & Negative & Negative & Negative & Negative & Negative & Negative \\
\hline Ketone & Negative & Negative & Negative & Negative & Negative & Negative \\
\hline Urobilinogen & Normal & Normal & Normal & Normal & Normal & Normal \\
\hline Bilirubin & Negative & Negative & Negative & Negative & Negative & Negative \\
\hline Erythrocytes & Negative & Negative & Negative & Negative & Negative & Negative \\
\hline Haemoglobin & Negative & Negative & Negative & Negative & Negative & Negative \\
\hline
\end{tabular}

Data ara mean \pm standard deviation $(n=6)$

The different superscript in the same column are statistically significant $(\mathrm{p}<0.05)$

SD $\quad$ : standard diet group

$\mathrm{NaD} \quad$ : high- $\mathrm{NaCl}$ diet group

PD : high- $\mathrm{NaCl}$ diet + captopril $(25 \mathrm{mg} / \mathrm{kg})$

NaDP1 : high-NaCl diet + propolis from Riau Archipelago (200 mg/ kg)

NaDP2 : high- $\mathrm{NaCl}$ diet + propolis from Lampung $(200 \mathrm{mg} / \mathrm{kg})$

$\mathrm{NaDP} 3 \quad$ : high- $\mathrm{NaCl}$ diet + propolis from South Sulawesi $(200 \mathrm{mg} / \mathrm{kg})$

\section{Table 6: Haematological analysis.}

\begin{tabular}{|c|c|c|c|c|c|c|}
\hline Parameters & SD & $\mathrm{NaD}$ & PD & NaDP1 & $\mathrm{NaDP} 2$ & NaDP3 \\
\hline WBC $(1000 / \mu \mathrm{L})$ & $4.9 \pm 1.8^{\mathrm{a}, \mathrm{b}}$ & $2.1 \pm 0.5^{\mathrm{b}}$ & $5.7 \pm 1.4^{\mathrm{a}}$ & $6.1 \pm 2.0^{\mathrm{a}}$ & $7.0 \pm 2.9^{\mathrm{a}}$ & $4.1 \pm 0.9^{\mathrm{a}, \mathrm{b}}$ \\
\hline $\operatorname{Lym}(1000 / \mu \mathrm{L})$ & $1.5 \pm 1.2^{\mathrm{b}}$ & $3.2 \pm 0.5^{\mathrm{a}, \mathrm{b}}$ & $4.1 \pm 1.2^{\mathrm{a}}$ & $4.5 \pm 1.7^{\mathrm{a}}$ & $4.8 \pm 2.0^{\mathrm{a}}$ & $2.6 \pm 0.5^{\mathrm{a}, \mathrm{b}}$ \\
\hline Mono $(1000 / \mu \mathrm{L})$ & $0.16 \pm 0.10^{\mathrm{a}, \mathrm{b}}$ & $0.10 \pm 0.05^{\mathrm{b}}$ & $0.15 \pm 0.05^{\mathrm{a}, \mathrm{b}}$ & $0.16 \pm 0.05^{\mathrm{a}, \mathrm{b}}$ & $0.23 \pm 0.12^{\mathrm{a}}$ & $0.12 \pm 0.04^{\mathrm{a}, \mathrm{b}}$ \\
\hline Gran $(1000 / \mu \mathrm{L})$ & $1.6 \pm 0.7^{\mathrm{a}, \mathrm{b}}$ & $0.6 \pm 0.2^{\mathrm{b}}$ & $1.5 \pm 0.3^{\mathrm{a}, \mathrm{b}}$ & $1.4 \pm 1.2^{\mathrm{a}, \mathrm{b}}$ & $2.0 \pm 1.2^{\mathrm{a}}$ & $1.3 \pm 0.5^{\mathrm{a}, \mathrm{b}}$ \\
\hline $\mathrm{RBC}(1000 / \mu \mathrm{L})$ & $7.1 \pm 1.5^{\mathrm{a}, \mathrm{b}}$ & $6.3 \pm 1.8^{b}$ & $7.7 \pm 0.6^{\mathrm{a}, \mathrm{b}}$ & $8.6 \pm 0.7^{\mathrm{a}}$ & $7.7 \pm 1.0^{\mathrm{a}, \mathrm{b}}$ & $8.0 \pm 0.8^{\mathrm{a}, \mathrm{b}}$ \\
\hline $\mathrm{HGB}(\mathrm{g} / \mathrm{dL})$ & $13,1 \pm 3.0^{\mathrm{a}, \mathrm{b}}$ & $11.45 \pm 3.4^{\mathrm{b}}$ & $13.7 \pm 1.2^{\mathrm{a}, \mathrm{b}}$ & $15.9 \pm 1.5^{\mathrm{a}}$ & $14.2 \pm 2.3^{\mathrm{a}, \mathrm{b}}$ & $14.5 \pm 1.1^{\mathrm{a}, \mathrm{b}}$ \\
\hline HCT (\%) & $40.9 \pm 8.7^{\mathrm{a}, \mathrm{b}}$ & $36.6 \pm 10.3^{b}$ & $42.8 \pm 3.5^{\mathrm{a}, \mathrm{b}}$ & $49.2 \pm 4.6^{\mathrm{a}}$ & $44.4 \pm 6.6^{\mathrm{a}, \mathrm{b}}$ & $45.4 \pm 3.5^{\mathrm{a}, \mathrm{b}}$ \\
\hline MCV (fL) & $57.9 \pm 0.9^{\mathrm{a}, \mathrm{b}}$ & $58.4 \pm 1.5^{\mathrm{a}}$ & $55.7 \pm 1.0^{\mathrm{b}}$ & $57.1 \pm 1.5^{\mathrm{a}, \mathrm{b}}$ & $57.5 \pm 1.1^{\mathrm{a}, \mathrm{b}}$ & $57.0 \pm 1.6^{\mathrm{a}, \mathrm{b}}$ \\
\hline $\mathrm{MCH}(\mathrm{pg})$ & $18.3 \pm 0.5^{\mathrm{a}}$ & $18.0 \pm 0.8^{\mathrm{a}}$ & $17.8 \pm 0.3^{\mathrm{a}}$ & $18.4 \pm 0.6^{\mathrm{a}}$ & $18.4 \pm 0.5^{\mathrm{a}}$ & $18.1 \pm 0.5^{\mathrm{a}}$ \\
\hline $\mathrm{MCHC}(\mathrm{g} / \mathrm{dL})$ & $31.8 \pm 0.7^{\mathrm{a}, \mathrm{b}}$ & $31.0 \pm 1.1^{\mathrm{b}}$ & $32.0 \pm 0.5^{\mathrm{a}, \mathrm{b}}$ & $32.2 \pm 0.4^{\mathrm{a}}$ & $32.0 \pm 0.5^{\mathrm{a}, \mathrm{b}}$ & $3.84 \pm 0.1^{\mathrm{a}, \mathrm{b}}$ \\
\hline RDW (\%) & $14.6 \pm 0.9^{\mathrm{a}}$ & $15.2 \pm 0.5^{\mathrm{a}}$ & $14.9 \pm 0.5^{\mathrm{a}}$ & $15.3 \pm 0.6^{\mathrm{a}}$ & $15.2 \pm 0.4^{\mathrm{a}}$ & $15.2 \pm 0.9^{\mathrm{a}}$ \\
\hline $\operatorname{PLT}(1000 / \mu \mathrm{L})$ & $808.1 \pm 430.9^{\mathrm{a}, \mathrm{b}}$ & $285.5 \pm 62.7^{\mathrm{b}}$ & $728.2 \pm 217.9^{\mathrm{a}, \mathrm{b}}$ & $1231.4 \pm 338.2^{\mathrm{a}}$ & $971.3 \pm 382.1^{\mathrm{a}}$ & $925.6 \pm 303.7+$ \\
\hline MPV (fL) & $5.9 \pm 0.5^{\mathrm{a}}$ & $6.0 \pm 0.3^{\mathrm{a}}$ & $5.8 \pm 0.3^{\mathrm{a}}$ & $5.7 \pm 0.4^{\mathrm{a}}$ & $6.0 \pm 0.3^{\mathrm{a}}$ & $5.6 \pm 0.1^{\mathrm{a}}$ \\
\hline PDW & $16.0 \pm 0.1^{\mathrm{a}}$ & $16.2 \pm 0.3^{\mathrm{a}}$ & $16.0 \pm 0.2^{\mathrm{a}}$ & $16.0 \pm 0.1^{\mathrm{a}}$ & $16.0 \pm 0.1^{\mathrm{a}}$ & $16.0 \pm 0.1^{\mathrm{a}}$ \\
\hline PCT (\%) & $0.4 \pm 0.2^{\mathrm{a}, \mathrm{b}}$ & $0.17 \pm 0.0^{\mathrm{b}}$ & $0.4 \pm 0.1^{\mathrm{a}, \mathrm{b}}$ & $0.6 \pm 0.0^{\mathrm{a}}$ & $0.5 \pm 0.2^{\mathrm{a}}$ & $0.5 \pm 0.2^{\mathrm{a}}$ \\
\hline
\end{tabular}

Data ara mean \pm standard deviation $(n=6)$

The different superscript in the same column are statistically significant $(\mathrm{p}<0.05)$

SD : standard diet group

$\mathrm{NaD} \quad:$ high- $\mathrm{NaCl}$ diet group

$\mathrm{PD} \quad$ : high-NaCl diet + captopril $(25 \mathrm{mg} / \mathrm{kg})$

NaDP1 : high-NaCl diet + propolis from Riau Archipelago (200 mg/ kg)

$\mathrm{NaDP} 2 \quad:$ high-NaCl diet + propolis from Lampung $(200 \mathrm{mg} / \mathrm{kg})$

$\mathrm{NaDP} 3 \quad$ : high- $\mathrm{NaCl}$ diet + propolis from South Sulawesi $(200 \mathrm{mg} / \mathrm{kg})$

\section{Table 7: Relative weight (\%) of organs.}

\begin{tabular}{|c|c|c|c|c|c|c|}
\hline Groups & Heart & Liver & Kidney & Spleen & Lungs & Urinary bladder \\
\hline Standard diet & $0.50 \pm 0.03^{\mathrm{a}}$ & $3.34 \pm 0.14^{\mathrm{a}}$ & $0.38 \pm 0.04^{\mathrm{a}}$ & $0.23 \pm 0.03^{\mathrm{a}}$ & $052 \pm 0.06^{\mathrm{a}}$ & $0.07 \pm 0.02^{\mathrm{a}}$ \\
\hline High- $\mathrm{NaCl}$ diet group & $0.44 \pm 0.03^{\mathrm{a}, \mathrm{b}}$ & $3.05 \pm 0.34^{\mathrm{a}}$ & $0.36 \pm 0.05^{\mathrm{a}}$ & $0.21 \pm 0.02^{\mathrm{a}}$ & $0.47 \pm 0.04^{\mathrm{a}}$ & $0.07 \pm 0.00^{\mathrm{a}}$ \\
\hline High- $\mathrm{NaCl}$ diet + captopril & $0.39 \pm 0.03^{\mathrm{b}}$ & $3.45 \pm 0.21^{\mathrm{a}}$ & $0.36 \pm 0.02^{\mathrm{a}}$ & $0.20 \pm 0.02^{\mathrm{a}}$ & $0.47 \pm 0.05^{\mathrm{a}}$ & $0.06 \pm 0.01^{\mathrm{a}}$ \\
\hline High- $\mathrm{NaCl}$ diet + propolis from Riau Archipelago & $0.40 \pm 0.02^{\mathrm{b}}$ & $3.20 \pm 0.15^{\mathrm{a}}$ & $0.36 \pm 0.02^{\mathrm{a}}$ & $0.20 \pm 0.04^{\mathrm{a}}$ & $0.46 \pm 0.02^{\mathrm{a}}$ & $0.06 \pm 0.01^{\mathrm{a}}$ \\
\hline High- $\mathrm{NaCl}$ diet + propolis from Lampung & $0.39 \pm 0.05^{\mathrm{b}}$ & $3.38 \pm 0.33^{\mathrm{a}}$ & $0.35 \pm 0.03^{\mathrm{a}}$ & $0.23 \pm 0.10^{\mathrm{a}}$ & $0.50 \pm 0.05^{\mathrm{a}}$ & $0.05 \pm 0.01^{\mathrm{a}}$ \\
\hline High- $\mathrm{NaCl}$ diet + propolis from South Sulawesi & $0.39 \pm 0.12^{\mathrm{b}}$ & $2.92 \pm 0.37^{a}$ & $0.33 \pm 0.02^{\mathrm{a}}$ & $0.21 \pm 0.03^{\mathrm{a}}$ & $0.48 \pm 0.08^{\mathrm{a}}$ & $0.06 \pm 0.02^{\mathrm{a}}$ \\
\hline
\end{tabular}

Data ara mean \pm standard deviation $(n=6)$

The different superscript in the same column are statistically significant $(\mathrm{p}<0.05)$ 
which reported that salt inhibited obesogenic effect of high fat diet by decreasing adipocytes size and leptin concentrations. ${ }^{38}$ In addition, we speculated that difference in $\mathrm{NaCl}$ concentration might be responsible for this result. The studies which showed obesogenic effect of high$\mathrm{NaCl}$ diet were not more than $4 \%{ }^{35-36}$ In contrast, our present study and the study of Pitynski-Mille et al. administered the diet with aroud $8 \%$ $\mathrm{NaCl}$. This concentration might cause too salty diet and could decrease food intake. ${ }^{39}$

The present study found that Indonesian propolis successfully decreased either SBP or DBP of hypertensive rats. Indeed, SBP was normalized after 1 week of propolis administration. Propolis is rich in phytochemical compounds, such as phenolics, flavonoids, and terpenes..$^{19}$ The prior studies also reported the antihypertensive activity of propolis from several regions, including Brazil, Australia, and Tunisia. ${ }^{21,40-43}$ Several compounds with antihypertensive activity have also been isolated from propolis, including dihydrokaempferide, kaempferide, butelelol, isosakuranetin, and caffeoylquinic acid. ${ }^{21,41}$ Indonesian propolis is a tropical-type propolis containing phenolic and flavonoid compounds and possesses strong antioxidant activity. ${ }^{20,44-45}$. The antioxidant activity of Indonesian propolis was proposed to underlie its antihypertensive activity through enhancing bioavailability of NO which responsible for vessel effect vasodilation. ${ }^{46}$ Furthermore, low antioxidant levels are associated with hypertension. ${ }^{47}$

We further analyzed the urine of rats after 1 week of intervention. We found some propolis samples including propolis from Riau Archipelago and Lampung had diuretic effect which might also be responsible for its antihypertensive effect. The evidences have showed the diuretic agents as promising antihypertension by removing any extra fluid and widening blood vessel. ${ }^{48}$ Unfortunately, we also found relatively higher concentrations of urine protein in all propolis groups, which in contrast to the current knowledge. ${ }^{49}$ However, one toxicological study reported Brazilian propolis induced acute renal failure ${ }^{50}$ Our present results were similar to antihypertensive effect of amlodipine. ${ }^{51-53}$ Therefore, our data suggest the possible mechanism of Indonesian propolis as antihypertension may be similar to the antihypertensive effect of calcium channel blockers (CCBs).

The other possible mechanism of propolis in lowering blood pressure is probably through lipid metabolism. Propolis from Lampung decreased significantly LDL levels compared to high- $\mathrm{NaCl}$ only group. We also found propolis from South Sulawesi increased significantly HDL levels when compared to standard diet group. The previous studies showed that propolis from Nigeria and Brazil could improve LDL and HDL levels. ${ }^{54-55}$ LDL levels have been reported to correlate with blood pressure in Japanese women that probably due to its association with leptin resistance. ${ }^{56}$ In addition, low HDL levels are also associated with reduced kidney function and may contribute to hypertension. ${ }^{57}$

The haematological parameters data mainly showed no significant changes between interventions and control group. Nevertheless, increased lymphocyte concentrations in propolis groups possibly were related to its immunomodulatory effect. ${ }^{58}$ In addition, our present study found relatively low concentrations of several haematological parameters in high- $\mathrm{NaCl}$ only group compared to propolis group(s). Increase in blood volume due to high- $\mathrm{NaCl}$ diet might cause relatively low concentration of several haematological parameters. ${ }^{59}$ In fact, the prior study reported protective effect of propolis on haematological alterations. ${ }^{60-61}$

Chronic high-salt diet has been found to alter weight of several organs which may indicate pathological changes. ${ }^{62}$ From the relative weight of organs, however, we did not find the possibility of alterations in liver, kidney, spleen, lungs, and urinary bladder. We found only decrease in relative weight of heart which might be attributed to hypertonic effect of $\mathrm{NaCl}$ in plasma result in decreased water contents in organs. ${ }^{63}$ However, changes in relative weight of organs are not always correlated with pathological condition. ${ }^{64}$

\section{CONCLUSION}

The present study found that high- $\mathrm{NaCl}$ diet did not cause weight gain. All propolis samples ameliorated high- $\mathrm{NaCl}$ diet-induced hypertension, where the biggest changes found in both group administered with propolis from Riau Archipelago and those with propolis from South Sulawesi. According to 24-h urine volume, propolis from Riau Archipelago and Lampung possessed diuretic effect. Increased urine protein was found in all propolis groups. Nevertheless, the other parameters (density, $\mathrm{pH}$, consistency, leukocytes, nitrite, glucose, ketone, urobilinogen, bilirubin, erythrocytes, and haemoglobin) were not affected. Propolis from Lampung and South Sulawesi are seemed to improve LDL and HDL concentrations, respectively. Moreover, haematological parameters mainly did not change after the treatments. In addition, we only found decrease in relative weight of liver in all groups administered with high- $\mathrm{NaCl}$ diet.

Our study suggests that the antihypertensive effect of Indonesian propolis samples through different pathways. Care should be taken in use of propolis among patients with proteinuria. Histopathological changes need further investigation.

\section{ACKNOWLEDGEMENT}

We wish to thank the Directorate of Research and Community Service, Directorate General for Research and Development, Ministry of Research and Higher Education of The Republic of Indonesia that funded this research (No. 4224/IT3.L1/PN/2019).

\section{REFERENCES}

1. Mills KT, Stefanescu A, \& He J. The global epidemiology of hypertension. Nature Reviews Nephrology. 2020; 2020(16): 223-237.

2. WHO.int. Hypertension. [Updated: 2019 September 13; cited; October 13, 2020]

3. Lastra G, Syed S, Kurukulasuriya LR, Manrique C, Sowers JR. Type 2 diabetes mellitus and hypertension: an update. Endocrinol Metab Clin. 2014; 43(1): 103122. doi: 10.1016/j.ecl.2013.09.005.

4. Lea JP, Nicholas SB. Diabetes mellitus and hypertension: key risk factors for kidney disease. J Natl Med Assoc. 2002; 94(8 Suppl), 7S.

5. KokuboY, Matsumoto, C. Hypertension is a risk factor for several types of heart disease: review of prospective studies. In Hypertension: from basic research to clinical practice. Cham: Springer International Publishing, p. 419-426; 2016.

6. Christakoudi S, Kakourou A, Markozannes G, Tzoulaki I, Weiderpass E, Brennan P. Blood pressure and risk of cancer in the European Prospective Investigation into Cancer and Nutrition. International Journal of Cancer. 2020; 146(10): 26802693. doi: 10.1002/ijc.32576.

7. Obisesan TO. Hypertension and cognitive function. Clin in Geriatr Med. 2009; 25(2): 259-288. doi: 10.1016/j.cger.2009.03.002.

8. Alcocer L, Cueto L. Hypertension, a health economics perspective. Therap Adv in Cardiovasc Dis. 2008; 2(3): 147-155. doi: 10.1177/ 1753944708090572.

9. Forman John P, Meir J. Stampfer, and Gary C. Curhan. Diet and lifestyle risk factors associated with incident hypertension in women. Jama 302.4. 2009; 401-411. doi: 10.1001/jama.2009.1060.

10. Roberts WC. High salt intake, its origins, its economic impact, and its effect on blood pressure. American Journal of Cardiology. 2001; 88(11): 1338-1346. doi: 10.1016/S0002-9149(01)02105-1.

11. Rust $P$, \& Ekmekcioglu C. Impact of salt intake on the pathogenesis and treatment of hypertension. In Hypertension: from basic research to clinical practice. Cham: Springer International Publishing, p. 61-84; 2016.

12. Karppanen $H$, Mervaala E. Sodium intake and hypertension. Progress in cardiovascular diseases. Prog Cardiovasc Dis. 2006; 49(2): 59-75. doi: 10.1016/j. pcad.2006.07.001.

13. Shimosawa T. Salt, the renin-angiotensin-aldosterone system and resistant hypertension. Hypertens Res. 2013; 36(8): 657-660. doi: 10.1038/hr.2013.69.

14. Caligiuri SP, Pierce GN. A review of the relative efficacy of dietary, nutritional supplements, lifestyle, and drug therapies in the management of hypertension. Critical reviews in food science and nutrition. 2017; 57(16): 3508-3527. doi: 10.1080/10408398.2016.1142420. 
15. Huang WY, Davidge ST, Wu J. Bioactive natural constituents from food sources potential use in hypertension prevention and treatment. Crit Rev Food Sci Nutr. 2013; 53(6): 615-630. doi: 10.1080/10408398.2010.550071.

16. Bankova V, Popova M, Bogdanov S, Sabatini AG. Chemical composition of European propolis: expected and unexpected results. Zeitschrift für Naturforschung C, 2002; 57(5-6): 530-533. doi: 10.1515/znc-2002-5-622.

17. Xuan $H$, Zhu $R$, Li Y, Hu F. Inhibitory effect of chinese propolis on phosphatidylcholine-specific phospholipase C activity in vascular endothelial cells. Evid Based Complement Alternat Med. 2011; 2011(1-2): 985278. doi: $10.1155 / 2011 / 985278$

18. Król W, Bankova V, Sforcin JM, Szliszka E, Czuba Z, Kuropatnicki AK. Propolis: properties, application, and its potential. Evid Based Complement Alternat Med. 2013; 2013: 807578. doi: 10.1155/2013/807578.

19. Huang S, Zhang CP, Wang K, Li GQ, Hu FL. Recent advances in the chemical composition of propolis. Molecules J. 2014; 19(12), 19610-19632. doi: 10.3390/ molecules 191219610

20. Fikri AM, Sulaeman A, Marliyati SA, Fahrudin M. Antioxidant activity and total phenolic content of stingless bee propolis from Indonesia. J Apicultural Science, 2019; 63(1): 139-147. doi: 10.2478/JAS-2019-0012.

21. Maruyama $H$, Sumitou $Y$, Sakamoto $T$, Araki $Y$, Hara $H$. Antihypertensive effects of flavonoids isolated from brazilian green propolis in spontaneously hypertensive rats. Biol Pharm Bull. 2009; 32(7): 1244-1250. doi: 10.1248/ bpb.32.1244

22. Hasan AEZ, Mangunwidjaja D, SunartiTC, Suparno O, Setiyono A. Investigating the antioxidant and anticytotoxic activities of propolis collected from five regions of Indonesia and their abilities to induce apoptosis. Emirate J Food \& Agric. 2014; 390-398. doi: 10.9755/ejfa.v26i5.16549.

23. Kalsum N, Sulaeman A, Setiawan B, Wibawan IWT. Preliminary studies of the immunomodulator effect of the propolis Trigona spp. extract in a mouse model. J Agric \& Veterinary Sci. 2017; 10: 75-80. doi: 10.9790/2380-1002027580.

24. Sahlan M, Devina A, Pratami DK, Situmorang H, Farida S, Munim A, et al. Antiinflammatory activity of Tetragronula species from Indonesia. Saudi J Bio Sci. 2019; 26(7): 1531-1538. doi: 10.1016/j.sjbs.2018.12.008.

25. Chen YF, Meng QC, Wyss JM, Jin H, Oparil, S. High- $\mathrm{NaCl}$ diet reduces hypothalamic norepinephrine turnover in hypertensive rats. Hypertension J. 1988; 11(1): 55-62. doi: 10.1161/01.HYP.11.1.55.

26. Yu HC, Burrell LM, Black MJ, Wu LL, Dilley RJ, Cooper ME, et al. Salt induces myocardial and renal fibrosis in normotensive and hypertensive rats. Circulation J. 1998; 93(23): 2621-2628. doi: 10.1161/01.cir.98.23.2621.

27. Aoki H, Nakata M, Dezaki K, Lu M, Gantulga D, Yamamoto K, et al. Ghrelin counteracts salt-induced hypertension via promoting diuresis and renal nitric oxide production in Dahl rats. Endocr J. 2013; 12-0371. doi: 10.1507/endocrj. ej12-0371.

28. Gogebakan A, Talas ZS, Ozdemir I, Sahna E. Role of propolis on tyrosine hydroxylase activity and blood pressure in nitric oxide synthase-inhibited hypertensive rats. Clin and Experiment Hypertension. 2012; 34(6): 424-428. doi: 10.3109/10641963.2012.665542.

29. Selamoglu Talas Z. Propolis reduces oxidative stress in L-NAME-induced hypertension rats. Cell biochem \& funct, 2014; 32(2): 150-154. doi: 10.1002/ cbf.2986.

30. Colli LG, Belardin LB, Echem C, Akamine EH, Antoniassi MP, Andretta RR, et al. Systemic arterial hypertension leads to decreased semen quality and alterations in the testicular microcirculation in rats. Scientific reports. 2019; 9(1): 1-12. doi : 10.1038/s41598-019-47157-w

31. Malkoff J. Non-invasive blood pressure for mice and rats. Animal Lab News, Kent Scientific Corporation. 2005; 1-12. doi: 10.1007/978-1-60761-247-6_3.

32. Purohit A, Joshi K, Kotru B, Kotru S. (2013). Effect of Indian propolis on haematological parameters in experimentally induced hyperlipidemic male albino rabbits. Asian J Pharm Clin Res. 2013; 6(1): 17-19.

33. Kotsis V, Stabouli S, Papakatsika S, Rizos Z, Parati G. Mechanisms of obesityinduced hypertension. Hypertension res. 2010; 33(5): 386-393. doi: 10.1038/ hr.2010.9.

34. Jiang SZ, Lu W, Zong XF, Ruan HY, Liu Y. Obesity and hypertension. Exp Ther Med. 2016; 12(4): 2395-2399. doi: 10.3892/etm.2016.3667.

35. Dobrian AD, Schriver SD, Lynch T, Prewitt RL. Effect of salt on hypertension and oxidative stress in a rat model of diet-induced obesity. American J Physio-Renal Physio. 2003; 285(4): F619-F628. doi: 10.1152/ajprenal.00388.2002.

36. Lanaspa MA, Kuwabara M, Andres-Hernando A, Li N, Cicerchi C, Jensen T. High salt intake causes leptin resistance and obesity in mice by stimulating endogenous fructose production and metabolism. Proc Natl Acad Sci U S A. 2018; 115(12), 3138-3143. doi: 10.1073/pnas.1713837115.

37. Contreras RJ. High- $\mathrm{NaCl}$ intake of rat dams alters maternal behavior and elevates blood pressure of adult offspring. Am J Physiol. 1993; 264(2): R296-R304. doi: 10.1152/ajpregu.1993.264.2.R296.
38. Pitynski-Miller D, Ross M, Schmill M, Schambow R, Fuller T, Flynn FW, Skinner DC. A high salt diet inhibits obesity and delays puberty in the female rat. Int $J$ Obes (Lond). 2017: 41(11): 1685-1692. doi: 10.1038/ijo.2017.154.

39. Bolhuis DP, Lakemond CM, de Wijk RA, Luning PA, de Graaf C. Both longer oral sensory exposure to and higher intensity of saltiness decrease ad libitum food intake in healthy normal-weight men. $\mathrm{J}$ of nutrition. 2011; 141(12): 2242-2248. doi:10.3945/jn.111.143867.

40. Kubota Y, Umegaki K, Kobayashi K, Tanaka N, Kagota S, Nakamura K, \& Shinozuka K. Anti-hypertensive effects of Brazilian propolis in spontaneously hypertensive rats. Clin Exp Pharmacol Physiol. 2004; 31: S29-S30. doi: 10.1111/j.1440-1681.2004.04113.x

41. Mishima S, Yoshida C, Akino S, Sakamoto T. Antihypertensive effects of Brazilian propolis: identification of caffeoylquinic acids as constituents involved in the hypotension in spontaneously hypertensive rats. Biol Pharm Bull. 2005; 28(10): 1909-1914. doi: 10.1248/bpb.28.1909.

42. Massaro FC, Brooks PR, Wallace HM, Nsengiyumva V, Narokai L, Russell FD. Effect of Australian propolis from stingless bees (Tetragonula carbonaria) on pre-contracted human and porcine isolated arteries. PLoS One. 2013; 8(11): e81297. doi: 10.1371/journal.pone.0081297.

43. Gargouri W, Osés SM, Fernández-Muiño MA, Sancho MT, Kechaou N Evaluation of bioactive compounds and biological activities of Tunisian propolis. LWT. 2019; 111: 328-336. doi: 10.1016/j.Iwt.2019.05.044.

44. Fikri AM, Popova M, Sulaeman A, Bankova V. Stingless bees and Mangifera indica: A close relationship?. Indian J Nat Prod Resour. 2020; 11(2): 130-134.

45. Pratami DK, Mun'im A, Sundowo A, Sahlan M. Phytochemical profile and antioxidant activity of propolis ethanolic extract from Tetragonula bee. Pharmacogn J. 2018: 10(1). doi: 10.5530/pj.2018.1.23.

46. Sinha N, Kumar Dabla P. Oxidative stress and antioxidants in hypertension-a current review. Curr hypertens rev. 2015; 11(2): 132-142. doi: 10.2174/1573402 111666150529130922

47. Vaziri ND. Roles of oxidative stress and antioxidant therapy in chronic kidney disease and hypertension. Curr Opin Nephrol Hypertens. 2004; 13(1): 93-99. doi: 10.1097/00041552-200401000-00013.

48. Roush GC, Sica DA. Diuretics for hypertension: a review and update. Am J Hypertens. 2016; 29(10): 1130-1137. doi: 10.1093/ajh/hpw030.

49. Xu R, Sun S, Huo Y, Yun L, Huang S, Li G, et al. Effects of ACEls versus ARBs on proteinuria or albuminuria in primary hypertension: a meta-analysis of randomized trials. Medicine J. 2015; 94(39). doi: 10.1097/MD.0000000000001560.

50. Li YJ, Lin JL, Yang CW, Yu CC. Acute renal failure induced by a Brazilian variety of propolis. Am J Kidn Dis. 2005; 46(6): e125-e129. doi: 10.1053/j. ajkd.2005.08.028.

51. Wright Jr JT, Bakris G, Greene T, Agodoa LY, Appel LJ, Charleston J, et al. Effect of blood pressure lowering and antihypertensive drug class on progression of hypertensive kidney disease: results from the AASK trial. JAMA. 2002; 288(19): 2421-2431. doi: 10.1001/jama.288.19.2421.

52. Praga M, Andrade CF, Luno J, Arias M, Poveda R., Mora J, et al. Antiproteinuric efficacy of losartan in comparison with amlodipine in non-diabetic proteinuric renal diseases: a double-blind, randomized clinical trial. Nephrol Dial Transplant. 2003; 18(9): 1806-1813. doi: 10.1093/ndt/gfg284.

53. Flynn ER, Marbury DC, Sawyer RT, Lee J, Teutsch C, Kauser K, et al Amlodipine reduces inflammation despite promoting albuminuria in the streptozotocin-induced diabetic rat. Nephron extra. 2012; 2(1): 205-218. doi: 10.1159/000339436.

54. El-Sayed ESM, Abo-Salem OM, Aly HA, Mansour AM. Potential antidiabetic and hypolipidemic effects of propolis extract in streptozotocin-induced diabetic rats. Pak J Pharm Sci. 2009; 22(2).

55. Abdulbasit A, Oladayo M, Olamide F, Olasile O, Babatunde I, Gbolahan B Effect of Nigerian propolis on glycemia, lipid profile, and oxidative stress markers in alloxan-induced diabetic rats. PHOL. 2013; 2: 149-158.

56. Oda E, Kawai R. LDL cholesterol is associated with blood pressure in Japanese women. Diabetes Care. 2009; 32(9): e113-e113.

57. Odden MC, Tager IB, Gansevoort RT, Bakker SJ, Fried LF, Newman AB, et al. Hypertension and low HDL cholesterol were associated with reduced kidney function across the age spectrum: a collaborative study. Ann Epidemiol. 2013; 23(3): 106-111. doi: 10.1016/j.annepidem.2012.12.004

58. Wolska K, Gorska A, Antosik K, Lugowska K. Immunomodulatory effects of Propolis and its components on basic immune cell functions. Indian J Pharm Sci. 2019; 81(4): 575-588. doi: 10.36468/pharmaceutical-sciences.548.

59. Gupta BN, Linden RJ, Mary DASG, Weatherill D. The influence of high and low sodium intake on blood volume in the dog. Q J Exp Physiol. 1981; 66(2): 117128. doi: 10.1113/expphysiol.1981.sp002539.

60. Yonar ME, Yonar SM, Ural MŞ, Silici S, Düşükcan M. Protective role of propolis in chlorpyrifos-induced changes in the haematological parameters and the oxidative/antioxidative status of Cyprinus carpio carpio. Food \& Chem Toxicol. 2012; 50(8): 2703-2708. doi: 10.1016/j.fct.2012.05.032. 
61. Yonar SM, Ural MŞ, Silici S, Yonar ME. Malathion-induced changes in the haematological profile, the immune response, and the oxidative/antioxidant status of Cyprinus carpio carpio: Protective role of propolis. Ecotoxicol Environ Saf. 2014;102: 202-209. doi: 10.1016/j.ecoenv.2014.01.007.

62. Tsunooka K, Morita $\mathrm{H}$. Effect of a chronic high-salt diet on whole-body and organ sodium contents of Dahl rats. J Hypertens. 1997; 15(8): 851-856. doi: 10.1097/00004872-199715080-00008.
63. Bybordi A, Tabatabaei SJ, Ahmadev A. Effect of salinity on the growth and peroxidase and IAA oxidase activities in canola. J Food Agric Environ. 2010; 8(1): 109-112.

64. Michael B, Yano B, Sellers RS, Perry R., Morton D, Roome N, et al. Evaluation of organ weights for rodent and non-rodent toxicity studies: a review of regulatory guidelines and a survey of current practices. Toxicol Pathol. 2007; 35(5): 742750. doi: 10.1080/01926230701595292.

\section{GRAPHICAL ABSTRACT}

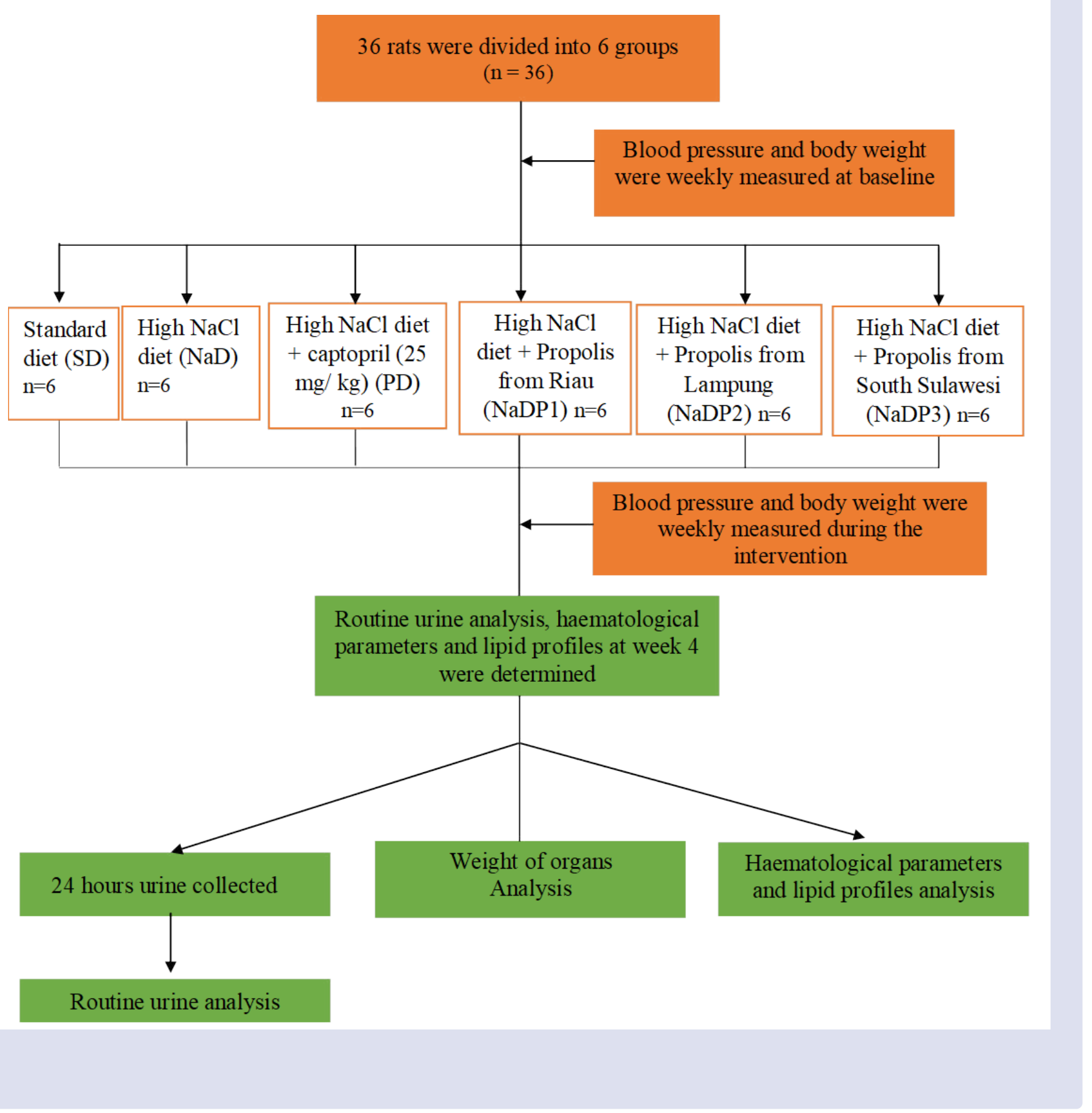




\section{SUMMARY}

- High- $\mathrm{NaCl}$ diet-induced hypertension on Sprague Dawley rats.

- Indonesian Propolis Shown antihypertensive effect through different pathways.

\section{ABOUT AUTHORS}

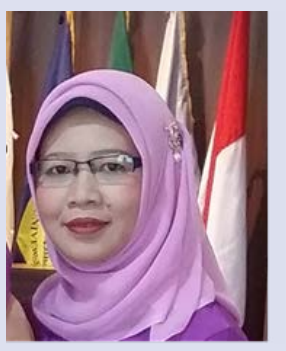

Ade Heri Mulyati, a Master at Department of Chemistry, Faculty of Mathematics and Natural Science, University of Pakuan Bogor and Doctoral student at Department of Community Nutrition, Faculty of Human Ecology, IPB University, Indonesia. She is expert in biochemistry, organic chemistry of natural materials, food chemistry and nutrition.

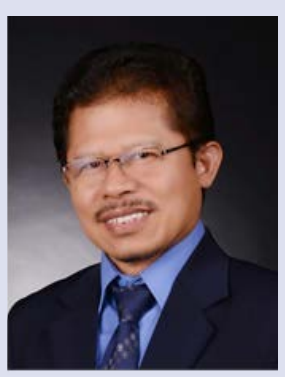

Ahmad Sulaeman, a professor of food safety and nutrition and head of Food Management and Environmental Health Division at Department of Community Nutrition Faculty of Human Ecology IPB University, Bogor Indonesia. His major research interest is in nutraceuticals especially in propolis and health. He also work on food product development for nutrition intervention.

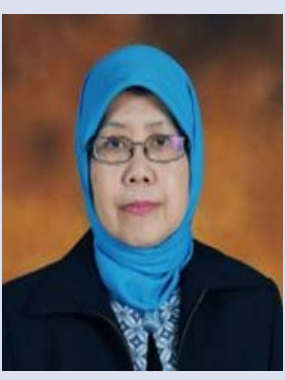

Sri Anna Marliyanti, a Professor at Department of Community Nutrition, Faculty of Human Ecology, IPB University, Indonesia. She is also the Head of the Department from 2018 until now. Her research areas include bioactive components, nutritious food formulation, functional food, food nutrification, fortification, and food intervention in the community.

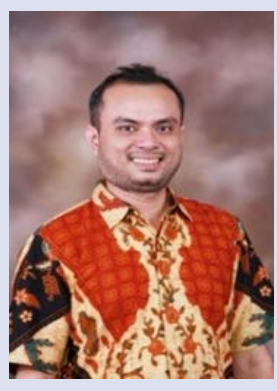

Mohamad Rafi, a Doctor at Department of Chemistry, Faculty of Mathematics and Natural Sciences, IPB University, Indonesia. His research interest in analytical chemistry, chemometrics, and metabolomics.

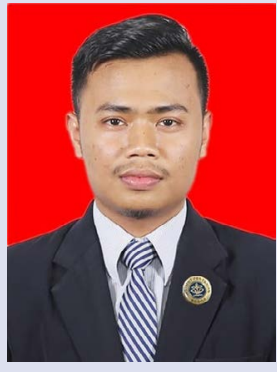

Al Mukhlas Fikri, A doctor in nutrition from IPB University. His research is mainly on nutrition and nutraceuticals.

Cite this article: Mulyati AH, Sulaeman A, Marliyati SA, Rafi M, Fikri AM. Preclinical Trial of Propolis Extract in Prevention of High Salt Diet-Induced Hypertension. Pharmacog J. 2021;13(1): 89-96. 